\title{
Macrófitas aquáticas do sistema lacustre do Vale do Rio Doce, Minas Gerais, Brasil ${ }^{1}$
}

\section{Aquatic macrophytes of the Rio Doce valley lacustrine system, Minas Gerais, Brazil}

\author{
Marco Otávio Pivari ${ }^{2,6}$, Vitor Baptista de Oliveira ${ }^{3}$, Flavio Magalhães Costa ${ }^{4}$ \\ Régis Moreira Ferreira ${ }^{5} \&$ Alexandre Salino ${ }^{2}$
}

\begin{abstract}
Resumo
Esta pesquisa trata da composição e da ocorrência de espécies de macrófitas aquáticas em área de proteção ambiental e áreas não protegidas, que compõem o conjunto de lagos do Vale do Rio Doce em Minas Gerais, terceiro maior sistema lacustre brasileiro. As informações foram levantadas a partir de publicações, material depositado em herbários e coletas botânicas entre os anos de 2007 e 2010, em ambientes aquáticos localizados no Parque Estadual do Rio Doce (PERD) e zona de amortecimento. Foram registradas 184 espécies pertencentes a distintos grupos taxonômicos, hábitos e formas biológicas, sendo aqui proposta a criação de uma nova categoria destas, designada embalsada, para contemplar plantas que se estabelecem em ilhas flutuantes. A pesquisa contribuiu com 152 novas citações para o Vale do Rio Doce em Minas Gerais, com dois primeiros registros nesse estado e com a descrição de uma espécie inédita para a ciência. A similaridade florística entre áreas protegidas e não protegidas indicou que o PERD guarda $74 \%$ das espécies de macrófitas aquáticas encontradas. Entretanto, 26\% do total de espécies estão desprotegidas, pois não ocorrem nessa unidade de conservação.
\end{abstract}

Palavras-chave: conservação, embalsada, ilha flutuante, plantas aquáticas.

\begin{abstract}
This paper refers to aquatic flora species composition and distribution in protected and surrounding areas located in the Rio Doce valley lakes, Minas Gerais state, the third largest Brazilian lacustrine system. Data were collected from previous works, herbaria collections and field samplings done between 2007 and 2010 in aquatic environments located within and around the Parque Estadual do Rio Doce (PERD). We recorded 184 aquatic macrophyte species in different taxonomic groups, habits and life forms. In this study we propose the creation of a new life-form category, "embalsada", applied to plants established on floating meadows. This work contributed 152 new records for the Rio Doce valley in Minas Gerais, two of which were new to the state and one undescribed species. A floristic similarity characterization between protected and surrounding areas indicated that PERD protects $74 \%$ of all aquatic macrophyte species found. However, we found that $26 \%$ of all species are unsafe because they do not occur in this conservation unit.
\end{abstract}

Key words: aquatic plants, conservation, "embalsada", floating meadow.

\section{Introdução}

O número de estudos envolvendo macrófitas aquáticas no Brasil é crescente, especialmente levando-se em consideração as duas últimas décadas, onde os principais motivos que contribuíram para esse cenário foram o interesse pela biodiversidade e a necessidade de manejo de ecossistemas aquáticos (Thomaz \& Bini 2003).

Segundo estes autores, há o predomínio de trabalhos abordando inventários de espécies, porém a quantidade destes ainda é insuficiente dada a amplitude do território brasileiro, cuja rede

\footnotetext{
'Parte da tese de Doutorado do primeiro autor.

${ }^{2}$ Universidade Federal de Minas Gerais, Instituto de Ciências Biológicas, Lab. Sistemática Vegetal, Av. Antônio Carlos 6627, 30130-000, Belo Horizonte, MG, Brasil. ${ }^{3}$ Centro Universitário do Leste de Minas Gerais, R. Bárbara Heliodora 725, 35160-215, Ipatinga, MG, Brasil.

${ }^{4}$ Instituto Nacional de Pesquisas da Amazônia, Av. André Araújo 2936, 69060-001, Manaus, AM, Brasil.

${ }^{5}$ Centro Universitário Belo Horizonte, Av. Mário Werneck 1685, 30455-610, Belo Horizonte, MG, Brasil.

${ }^{6}$ Autor para correspondência: marcopivari@yahoo.com.br
} 
hidrográfica é a maior do mundo. Enquanto isso, ambientes aquáticos naturais vem sendo alterados por processos de desmatamento, drenagem, poluição, construção de barragens ou canais de transposição, dentre outros.

A criação de áreas de preservação em território nacional não acompanha a velocidade da perda de habitats aquáticos. Segundo Medeiros (2006), no Brasil entre o período de 1993 a 2008, apenas oito áreas úmidas foram reconhecidas como Sítios Ramsar (SMASP 1997). Tais sítios representam, internacionalmente, locais de conservação da biodiversidade, além dos fatores econômico, cultural, científico e recreativo ligados aos recursos hídricos.

No estado de Minas Gerais, o conjunto de ambientes lacustres do Vale do Rio Doce apresentase, ao nível mundial, como peculiar refúgio da vida silvestre justificando sua inclusão no grupo de Sítios Ramsar, ocorrida no ano de 2009. Porém, segundo os critérios utilizados para tal reconhecimento, menos de um terço desses corpos d'água (42 lagoas inseridas no Parque Estadual do Rio Doce - PERD) foi contemplado, fato que mantém a maioria restante sob os impactos causados por diversas atividades humanas, comuns na zona de amortecimento do PERD. Destas, podem ser destacadas o cultivo de eucalipto em larga escala, a introdução de espécies exóticas (flora e fauna), poluição, criação de estradas não pavimentadas às margens de lagoas, além de caça e pesca predatória.

O Vale do Rio Doce mineiro passou a ser alvo de estudos de diversas áreas da limnologia, especialmente a partir de 1980. Foram desenvolvidas pesquisas nesse conjunto lacustre sobre geomorfologia, produção primária, dinâmica de nutrientes, organismos planctônicos e bentônicos, sedimentação e ictiologia (Saijo \& Tundisi 1985, 1989), às vezes relacionando características dos ambientes aquáticos aos impactos oriundos de atividades humanas (Maia-Barbosa et al. 2010). Entretanto, há carência de estudos sobre as macrófitas aquáticas (Esteves 1988), cujas informações atuais são escassas, até mesmo sobre a diversidade de espécies. De uma forma geral, essa também é a situação observada para todo o estado de Minas Gerais que, no período de 1985 a 2000, teve somente nove trechos das treze bacias hidrográficas contempladas com inventários da flora aquática, não permitindo extrapolações em termos da conservação e manejo desses organismos (Pedralli 2000).

Os objetivos do presente trabalho consistem em revelar a composição da flora presente no sistema lacustre do Vale do Rio Doce e caracterizar a ocorrência dessa comunidade na região, com base na presença das espécies em áreas de proteção ambiental, localizadas no Parque Estadual do Rio Doce (Sítio Ramsar), e em áreas não protegidas, na sua zona de amortecimento.

\section{Material e Métodos}

O sistema lacustre do Vale do Rio Doce (Fig. 1), localizado ao leste do estado de Minas Gerais $\left(19^{\circ} 29^{\prime} \mathrm{S}-19^{\circ} 49^{\prime} \mathrm{S}, 42^{\circ} 28^{\prime} \mathrm{W}-42^{\circ} 38^{\prime} \mathrm{W}\right)$, figura como o terceiro maior conjunto de lagos do território brasileiro (Tundisi et al. 1981). Fazem parte desse sistema cerca de 150 corpos d'água lênticos, representados majoritariamente por lagoas e um número menor de brejos. Segundo Esteves (1988), os mesmos foram originados no Pleistoceno através da barragem natural da desembocadura dos antigos afluentes dos rios da região, o que não é observado em nenhum outro local do Brasil. A principal causa da atual diferença de nível (20-50 m) entre as lagoas e os leitos dos rios Doce e Piracicaba deve-se a sucessivos períodos de intensa pluviosidade e estiagem, que provocaram fases de erosão e deposição de sedimentos. Esse fato explica a perenidade das lagoas, além das suas formas dendríticas, bem como a ausência de pulsos de inundação como descritos por Junk \& Silva (1999).

A elaboração do inventário das espécies da flora presentes no sistema lacustre do Vale do Rio Doce seguiu diferentes etapas, que envolveram a revisão de identificações provenientes de pesquisas anteriores (Pivari et al. 2008b), visitas aos herbários onde há registros para a área de estudo (BHCB, CESJ, HUNL, PERD e VIC) e coletas de material botânico in situ. Para realização destas, o mosaico composto por aproximadamente 150 lagoas e brejos foi subdividido visando padronizar espacialmente o esforço amostral. Dessa forma, durante o período de janeiro de 2007 a setembro de 2010 foram percorridos mensalmente 72 ambientes aquáticos, o que corresponde a cerca de $1.200 \mathrm{~km}^{2}$ inseridos nos diferentes locais do Parque Estadual do Rio Doce e zona de amortecimento. Os limites marginais das lagoas e brejos foram definidos incluindo-se a faixa de alagamento dos mesmos durante a estação chuvosa, a qual coincide com o início da vegetação ciliar (floresta estacional semidecidual) estabelecida em terreno não alagável.

As amostragens foram efetuadas por meio de caminhadas e com o auxílio de embarcações motorizadas ou caiaques, além de serem realizados mergulhos ou utilizadas hastes com ganchos para 
Sistema Lacustre do Vale do Rio Doce, MG

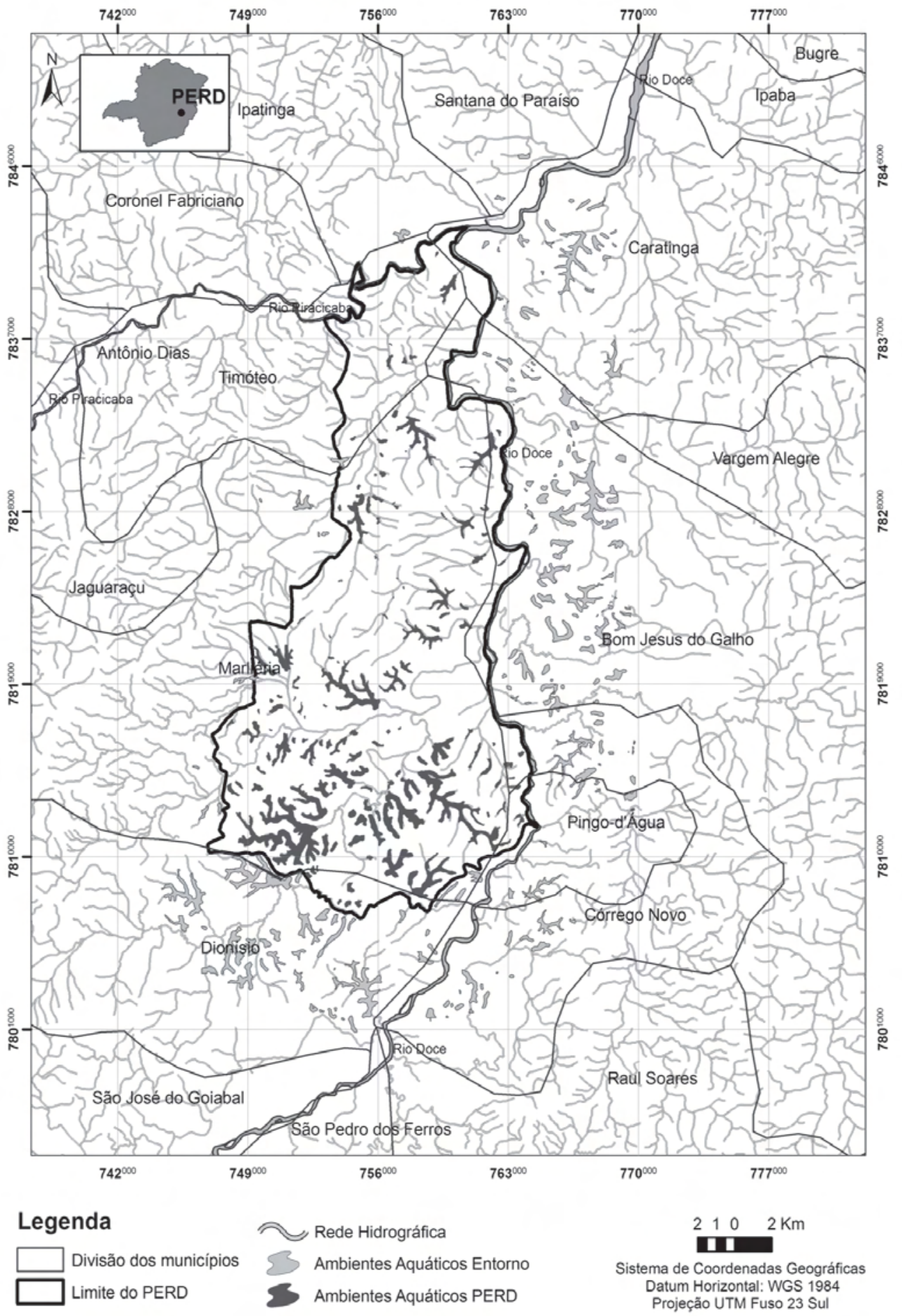

Figura 1 - Localização dos ambientes aquáticos (lagoas e brejos) do sistema lacustre do Vale do Rio Doce, Minas Gerais, destacando o Parque Estadual do Rio Doce (PERD).

Figure 1 - Aquatic environments (lakes and swamps) position at the Rio Doce valley lacustrine system, Minas Gerais, with emphasis on the Parque Estadual do Rio Doce (PERD). Following the sequence as shown in the figure: Municipalities boundaries, PERD boundary, Hydrographic network, Surrounding Aquatic Environments, PERD Aquatic Environments, Geographic Coordinates System, Horizontal Datum: WGS 1984, and UTM. 
averiguação de espécimes submersos em locais profundos. A partir disso, indivíduos em estado fértil foram coletados e processados segundo técnicas de herborização de plantas aquáticas (Haynes 1984; Ceska \& Ceska 1986; Pedralli 1990). A identificação dos táxons foi feita através de consultas à bibliografia especializada e por comparação morfológica com material depositado em herbários. Por fim, duplicatas foram enviadas a especialistas para confirmação ou determinação das identificações. As amostras obtidas encontram-se tombadas nos acervos dos herbários BHCB, CESJ, CGMS (HMS) e PERD.

A lista de espécies foi redigida seguindo o sistema de classificação de APG III (2009) para angiospermas, Kramer \& Green (1990) para pteridófitas e Raven et al. (1996) para carófitas. A terminologia nomenclatural e abreviações dos nomes dos autores seguiram Brummit \& Powell (1992), enquanto as formas biológicas foram consideradas segundo a classificação de Irgang et al. (1984) e Pedralli (1990), acrescida da terminologia embalsada (Neiff 1982), para as macrófitas aquáticas estabelecidas em ilhas flutuantes. Quando aplicável, uma mesma espécie foi enquadrada em mais de uma forma biológica.

Para quantificar a similaridade florística (diversidade Beta) entre o conjunto de ambientes aquáticos inseridos no PERD e o localizado na zona de amortecimento dessa unidade de conservação, foi calculado o coeficiente de similaridade, obtido pelo índice de Jaccard $\left(\mathrm{S}_{\mathrm{j}}\right)$. Essa abordagem foi realizada para caracterizar a ocorrência das espécies e inferir sobre o atual grau de conservação da comunidade de macrófitas aquáticas no sistema lacustre do Vale do Rio Doce, considerando que apenas uma parcela das lagoas e brejos é protegida pelo PERD.

\section{Resultados e Discussão}

O levantamento da flora presente no conjunto de lagoas e brejos do Vale do Rio Doce em Minas Gerais revelou a ocorrência de 184 espécies, enquadradas em diferentes grupos incluindo carófitas, pteridófitas e angiospermas, este último com predomínio em relação ao número de táxons (Tab.1). O total de gêneros registrado foi 121, dos quais destacaram-se Ludwigia L. e Cyperus L. com onze e nove espécies, respectivamente, enquanto das 64 famílias amostradas, Cyperaceae (26 spp.), Poaceae (17), Asteraceae (15) e Onagraceae (11) foram as mais representativas. Espécies de hábito herbáceo foram mais comuns (77\%), seguidos de arbustos (13\%), lianas (6\%) e árvores (4\%).
O número de espécies pode ser considerado alto para inventários enfocando macrófitas aquáticas por se tratar de uma área de estudo formada por um conjunto de ambientes aquáticos com características similares, ocupando no total cerca de $2.000 \mathrm{~km}^{2}$. No Vale do Rio Doce em Minas Gerais, os corpos d'água caracterizam-se por serem perenes e lênticos, com baixa flutuação no nível da água, por apresentarem solos argilosos e por não possuírem influência de pulsos de inundação com consequente comunicação superficial (Junk \& Silva 1999). No caso do Pantanal, por exemplo, uma área úmida expressivamente maior alcançando $110.000 \mathrm{~km}^{2}$ na estação chuvosa, Pott \& Pott (2000) registraram um número aproximado de macrófitas aquáticas (246 espécies). Além do tamanho da área de estudo, essa diferença pode ser atribuída ao fato do Pantanal apresentar ambientes aquáticos heterogêneos, variáveis quanto à sazonalidade, tipo de solo e velocidade da água, dentre outros parâmetros não observados no sistema lacustre do Vale do Rio Doce, os quais possibilitam o estabelecimento de maior diversidade de espécies devido à maior amplitude de condições (Thomaz 2006).

O presente estudo contribuiu com dados relevantes para o conhecimento da diversidade vegetal aquática por abordar uma expressiva área úmida brasileira, até então pouco explorada em termos florísticos. Como resultado dessa investigação, foram relatadas 152 primeiras citações de espécies para o Vale do Rio Doce em Minas Gerais e duas novas ocorrências para esse estado (Ceratopteris thalictroides e Habenaria nabucoi). Além disso, a pesquisa colaborou com a descrição de uma espécie: Lepidaploa opposita (Asteraceae), coletada nos domínios do Parque Estadual do Rio Doce (Teles et al. 2010).

Dentre as espécies que compõem a atual listagem predominaram aquelas consideradas com ampla distribuição geográfica: $37 \%$ nos neotrópicos, $20 \%$ em países da América do Sul, $17 \%$ cosmopolitas e $10 \%$ em regiões tropicais. Os demais $14 \%$ correspondem às espécies de ocorrência mais restrita, mencionada para diferentes regiões do Brasil, com exceção a Lepidaploa opposita, registrada apenas para a Mata Atlântica do sudeste (Teles et al. 2010). Constatou-se também Nymphaea caerulea, espécie introduzida da África atualmente subespontânea no centro-oeste e sudeste brasileiro (Amaral et al. 2008).

No geral, táxons referentes a plantas aquáticas apresentam ampla distribuição geográfica, a qual é atribuída a características relacionadas à eficiente ocupação de áreas alagáveis, incluindo ampla 
Tabela 1 - Espécies de macrófitas aquáticas do sistema lacustre do Vale do Rio Doce, Minas Gerais. Formas biológicas: A- anfíbia; Eb- embalsada; Em- emergente; Ep- epífita; FF- flutuante fixa; FL- flutuante livre; SF- submersa fixa; SL- submersa livre. Sistema lacustre: 1- espécie ocorrente no Parque Estadual do Rio Doce (PERD); 2- espécie ocorrente na Zona de Amortecimento PERD. Voucher: Herbário BHCB, exceto quando precedido por outra sigla. * Família pertencente a Carophyta; ** famílias pertencentes a Pteridophyta; demais famílias pertencentes a Angiospermae. Table 1 - Aquatic macrophyte species of the Rio Doce valley lacustrine system, Minas Gerais. Life forms: A- amphibian, Eb"embalsada", Em- emerging, Ep- epiphyte, FF- fixed floating, FL- free floating, SF- fixed submerged and SL- free submerged. Lacustrine system: species founded in the Parque Estadual do Rio Doce (PERD); 2- species founded in the PERD surrounding areas. Voucher: BHCB Herbarium, except when preceded by another initials. * Carophyta families; ** Pteridophyta families; another families belonging to Angiospermae.

\begin{tabular}{|c|c|c|c|c|}
\hline Grupo taxonômico & $\begin{array}{c}\text { Forma } \\
\text { biológica }\end{array}$ & Hábito & $\begin{array}{l}\text { Sistema } \\
\text { lacustre }\end{array}$ & Voucher \\
\hline \multicolumn{5}{|l|}{ CHARACEAE * } \\
\hline Chara guairensis R. Bicudo & $\mathrm{SF}$ & erva & 1,2 & 121679 \\
\hline Nitella cf. cernua A. Braun & $\mathrm{SF}$ & erva & 1 & 121759 \\
\hline \multicolumn{5}{|l|}{ BLECHNACEAE $* *$} \\
\hline Blechnum serrulatum Rich. & $\mathrm{Eb}$ & erva & 1,2 & 121697 \\
\hline \multicolumn{4}{|l|}{ LOMARIOPSIDACEAE $* *$} & 121600 \\
\hline \multicolumn{5}{|l|}{ LYCOPODIACEAE $* *$} \\
\hline \multicolumn{5}{|l|}{ OSMUNDACEAE $* *$} \\
\hline Osmunda regalis $\mathrm{L}$. & $\mathrm{Eb}$ & erva & 1 & 121718 \\
\hline \multicolumn{5}{|l|}{ PTERIDACEAE $* *$} \\
\hline Acrostichum danaeifolium Langsd. \& Fisch. & $\mathrm{Eb}$ & erva & 1,2 & 121686 \\
\hline Ceratopteris thalictroides $(\mathrm{L}$.$) Brongn.$ & Em & erva & 1,2 & 127403 \\
\hline Pityrogramma calomelanos (L.) Link & $\mathrm{A} / \mathrm{Eb}$ & erva & 1,2 & 121584 \\
\hline \multicolumn{5}{|l|}{ SALVINIACEAE $* *$} \\
\hline Salvinia auriculata Aubl. & FL & erva & 1,2 & 121760 \\
\hline \multicolumn{5}{|l|}{ THELYPTERIDACEAE $* *$} \\
\hline Thelypteris interrupta (Willd.) K. Iwats. & $\mathrm{A} / \mathrm{Eb}$ & erva & 1,2 & 121629 \\
\hline T. salzmannii (Fee) C.V. Morton & A & erva & 2 & 127374 \\
\hline \multicolumn{5}{|l|}{ ACANTHACEAE } \\
\hline Hygrophila costata Nees & A & erva & 2 & PERD 1037 \\
\hline Ruellia sp. & $\mathrm{Eb}$ & erva & 1 & 121875 \\
\hline \multicolumn{5}{|l|}{ ALISMATACEAE } \\
\hline Echinodorus bolivianus (Rusby) Holm-Niels. & A & erva & 1,2 & 121876 \\
\hline E. macrophyllus (Kunth) Micheli & $\mathrm{Eb}$ & erva & 2 & 43035 \\
\hline Sagittaria rhombifolia Cham. & Em & erva & 1 & 121765 \\
\hline \multicolumn{4}{|l|}{ ANACARDIACEAE } & 121596 \\
\hline \multicolumn{5}{|l|}{ ANNONACEAE } \\
\hline Annona glabra $\mathrm{L}$. & Em & árvore & 1,2 & 121548 \\
\hline Xylopia emarginata $\mathrm{L}$. & Em & árvore & 1,2 & 121849 \\
\hline \multicolumn{5}{|l|}{ APOCYNACEAE } \\
\hline Oxypetalum alpinum (Vell.) Fontella \& E.A. Schwarz & $\mathrm{A} / \mathrm{Eb}$ & liana & 1,2 & 121531 \\
\hline \multicolumn{5}{|l|}{ AQUIFOLIACEAE } \\
\hline Ilex affinis Gardner & Em & árvore & 1,2 & 121850 \\
\hline \multicolumn{5}{|l|}{ ARACEAE } \\
\hline Wolffiella oblonga (Phil.) Hegelm. & FL & erva & 1 & 133200 \\
\hline
\end{tabular}




\begin{tabular}{|c|c|c|c|c|}
\hline Grupo taxonômico & $\begin{array}{c}\text { Forma } \\
\text { biológica }\end{array}$ & Hábito & $\begin{array}{l}\text { Sistema } \\
\text { lacustre }\end{array}$ & Voucher \\
\hline \multicolumn{5}{|l|}{ ARALIACEAE } \\
\hline Centella asiatica (L.) Urb. & A & erva & 1,2 & 121527 \\
\hline Hydrocotyle leucocephala Cham. \& Schltdl. & A & erva & 2 & CESJ 40742 \\
\hline \multicolumn{5}{|l|}{ ARECACEAE } \\
\hline Bactris setosa Mart. & A & erva & 2 & CGMS 9565 \\
\hline \multicolumn{5}{|l|}{ ASTERACEAE } \\
\hline Adenostemma involucratum R.M. King \& H. Rob. & A & erva & 1 & 121743 \\
\hline Baccharis cf. glutinosa Pers. & A & erva & 1,2 & 121624 \\
\hline Conocliniopsis prasiifolia (DC.) R.M.King \& H. Rob. & $\mathrm{A} / \mathrm{Eb}$ & erva & 1,2 & 121592 \\
\hline Conyza sp. & A & erva & 1 & 127333 \\
\hline Cyrtocymura scorpioides (Lam.) H. Rob. & $\mathrm{Eb}$ & erva & 1,2 & 121590 \\
\hline Eclipta prostrata $(\mathrm{L}.) \mathrm{L}$. & $\mathrm{A} / \mathrm{Eb}$ & erva & 1,2 & 121580 \\
\hline Erechtites hieraciifolius (L.) Raf. ex DC. & A & erva & 1,2 & 121775 \\
\hline E. valerianifolius (Wolf) DC. & $\mathrm{A} / \mathrm{Eb}$ & erva & 1,2 & 121623 \\
\hline Lepidaploa opposita A. Teles, Sobral \& J.N. Nakaj. & A & erva & 1 & 32833 \\
\hline Leptostelma maximum D. Don & $\mathrm{Eb}$ & arbusto & 2 & 116535 \\
\hline Mikania cordifolia (L. f.) Willd. & $\mathrm{Eb}$ & liana & 1 & 131688 \\
\hline Pluchea sagittalis (Lam.) Cabrera & A & erva & 1,2 & 121627 \\
\hline Sphagneticola trilobata (L.) Pruski & A & erva & 1,2 & 121647 \\
\hline Struchium sparganophorum (L.) Kuntze & A & erva & 1 & PERD 1039 \\
\hline Vernonanthura brasiliana (L.) H. Rob. & $\mathrm{Eb}$ & arbusto & 1,2 & 121576 \\
\hline \multicolumn{5}{|l|}{ BEGONIACEAE } \\
\hline Begonia cucullata Willd. & $\mathrm{Em} / \mathrm{Eb}$ & erva & 1,2 & 127412 \\
\hline B. fischeri Schrank & $\mathrm{Em} / \mathrm{Eb}$ & erva & 1,2 & 116539 \\
\hline \multicolumn{5}{|l|}{ BURMANNIACEAE } \\
\hline Dictyostega orobanchoides (Hooker) Miers. & $\mathrm{Eb}$ & erva & 2 & 116540 \\
\hline \multicolumn{5}{|l|}{ CABOMBACEAE } \\
\hline Cabomba furcata Schult. \& Schult. f. & SF & erva & 1,2 & 127370 \\
\hline \multicolumn{5}{|l|}{ CAMPANULACEAE } \\
\hline Lobelia fistulosa Raf. & $\mathrm{Eb}$ & arbusto & 2 & 121579 \\
\hline \multicolumn{5}{|l|}{ CLEOMACEAE } \\
\hline Cleome aculeata $\mathrm{L}$. & A & erva & 1,2 & 121632 \\
\hline \multicolumn{5}{|l|}{ COMBRETACEAE } \\
\hline Combretum laxum Jacq. & $\mathrm{Eb}$ & liana & 1 & 121840 \\
\hline \multicolumn{5}{|l|}{ CORDIACEAE } \\
\hline Cordia bifurcata Roem. \& Schult. & $\mathrm{Eb}$ & erva & 2 & 121680 \\
\hline \multicolumn{5}{|l|}{ COMMELINACEAE } \\
\hline Commelina obliqua $\mathrm{Vahl}$ & $\mathrm{Eb}$ & erva & 1,2 & 121597 \\
\hline Commelina sp. & A & erva & 1,2 & 121776 \\
\hline \multicolumn{5}{|l|}{ COSTACEAE } \\
\hline Costus spiralis (Jacq.) Roscoe & $\mathrm{Eb}$ & erva & 1,2 & 121809 \\
\hline \multicolumn{5}{|l|}{ CUCURBITACEAE } \\
\hline Melothria fluminensis Gardner & $\mathrm{A} / \mathrm{Eb}$ & liana & 2 & 121526 \\
\hline \multicolumn{5}{|l|}{ CYPERACEAE } \\
\hline Cyperus giganteus Vahl & Em & erva & 2 & 121581 \\
\hline C. haspan $\mathrm{L}$. & $\mathrm{A} / \mathrm{Eb}$ & erva & 1,2 & 121538 \\
\hline C. iria $\mathrm{L}$. & A & erva & 2 & 127354 \\
\hline C. luzulae (L.) Rottb. ex Retz. & A & erva & 2 & 127394 \\
\hline
\end{tabular}




\begin{tabular}{|c|c|c|c|c|}
\hline Grupo taxonômico & $\begin{array}{c}\text { Forma } \\
\text { biológica }\end{array}$ & Hábito & $\begin{array}{l}\text { Sistema } \\
\text { lacustre }\end{array}$ & Voucher \\
\hline C. cf. mundtii (Nees) Kunth & A & erva & 1,2 & 127356 \\
\hline C. odoratus L. & A & erva & 1,2 & 121552 \\
\hline C. cf. rigens C. Presl & $\mathrm{Eb}$ & erva & 2 & 121898 \\
\hline C. surinamensis Rottb. & A & erva & 1,2 & 121529 \\
\hline Cyperus sp. & A & erva & 2 & 121544 \\
\hline Eleocharis geniculata (L.) Roem. \& Schltdl. & Em & erva & 1 & 121715 \\
\hline E. interstincta (Vahl) Roem. \& Schult. & Em & erva & 1,2 & 121571 \\
\hline E. minima Kunth & $\mathrm{A} / \mathrm{Eb}$ & erva & 1,2 & 121705 \\
\hline E. cf. nudipes (Kunth) Palla & $\mathrm{Eb}$ & erva & 1,2 & 131355 \\
\hline E. plicarhachis (Griseb.) Svenson & $\mathrm{Eb}$ & erva & 1,2 & 121637 \\
\hline Fimbristylis cf. spadicea (L.) Vahl & A & erva & 2 & 121620 \\
\hline Fuirena umbellata Rottb. & $\mathrm{Em} / \mathrm{Eb}$ & erva & 1,2 & 121574 \\
\hline Kyllinga pumila Michx. & A & erva & 1,2 & 121622 \\
\hline Oxycaryum cubense (Poepp. \& Kunth) Palla & $\mathrm{Eb} / \mathrm{Ep}$ & erva & 1,2 & 121568 \\
\hline Rhynchospora amazonica Poepp. \& Kunth & $\mathrm{Em}$ & erva & 2 & 121329 \\
\hline R. corymbosa $(\mathrm{L}$.$) Britton$ & $\mathrm{Em} / \mathrm{Eb}$ & erva & 1,2 & 121575 \\
\hline R. holoschoenoides (Rich.) Herter & A & erva & 1,2 & 133213 \\
\hline R. rugosa $($ Vahl) Gale & $\mathrm{Eb}$ & erva & 1 & 121830 \\
\hline R. trispicata (Nees) Steud. & Em & erva & 2 & 131329 \\
\hline Rhynchospora sp. & Em & erva & 2 & 131339 \\
\hline Scleria melaleuca Rchb. Ex Schltdl. \& Cham. & $\mathrm{A} / \mathrm{Eb}$ & erva & 1,2 & 121556 \\
\hline S. cf. mitis Bergius & A & erva & 1,2 & 121535 \\
\hline \multicolumn{5}{|l|}{ EUPHORBIACEAE } \\
\hline Caperonia palustris (L.) A. St.-Hil. & $\mathrm{A} / \mathrm{Eb}$ & erva & 1,2 & 121699 \\
\hline \multicolumn{5}{|l|}{ FABACEAE } \\
\hline Aeschynomene evenia $\mathrm{C}$. Wrignt \& Sauvalle & A & arbusto & 1 & 127355 \\
\hline A. fluminensis Vell. & Em & arbusto & 1,2 & 126502 \\
\hline A. sensitiva $\mathrm{Sw}$ & $\mathrm{A} / \mathrm{Eb}$ & arbusto & 1,2 & 121561 \\
\hline Dioclea violacea Mart. ex Benth. & A & liana & 1,2 & 131710 \\
\hline D. virgata (Rich.) Amshoff & $\mathrm{Eb}$ & liana & 1,2 & 121685 \\
\hline Vigna lasiocarpa (Mart. ex Benth.) Verdc. & $\mathrm{Eb}$ & liana & 1,2 & 121833 \\
\hline Vigna sp. & $\mathrm{Eb}$ & liana & 2 & 121545 \\
\hline \multicolumn{5}{|l|}{ GENTIANACEAE } \\
\hline Irlbachia alata (Aubl.) Maas & A & arbusto & 1,2 & 121599 \\
\hline \multicolumn{5}{|l|}{ GESNERIACEAE } \\
\hline Sinningia elatior (Kunth) Chautmes & $\mathrm{Eb}$ & arbusto & 2 & 116536 \\
\hline \multicolumn{5}{|l|}{ HALORAGACEAE } \\
\hline Myriophyllum aquaticum (Vell.) Verdc. & Em & erva & 2 & PERD 1038 \\
\hline \multicolumn{5}{|l|}{ HYDROCHARITACEAE } \\
\hline Apalanthe granatensis (Humb. \& Bonpl.) Planch. & $\mathrm{SF}$ & erva & 1 & 32871 \\
\hline Najas conferta (A. Braun) A. Braun & $\mathrm{SF}$ & erva & 1 & 31912 \\
\hline \multicolumn{5}{|l|}{ HYDROLEACEAE } \\
\hline Hydrolea spinosa $\mathrm{L}$. & A & arbusto & 2 & 116547 \\
\hline \multicolumn{5}{|l|}{ LAMIACEAE } \\
\hline Hyptis brevipes Poit. & $\mathrm{Eb}$ & erva & 2 & 121578 \\
\hline H. cf. fasciculata Benth. & $\mathrm{A} / \mathrm{Eb}$ & erva & 1,2 & 121618 \\
\hline H. cf. lorentziana O. Hoffm. & A & erva & 1,2 & 121723 \\
\hline H. microphylla Pohl ex Benth. & A & arbusto & 1,2 & 121730 \\
\hline H. pectinata (L.) Poit. & $\mathrm{A} / \mathrm{Eb}$ & arbusto & 2 & 121554 \\
\hline
\end{tabular}




\begin{tabular}{|c|c|c|c|c|}
\hline Grupo taxonômico & $\begin{array}{c}\text { Forma } \\
\text { biológica }\end{array}$ & Hábito & $\begin{array}{l}\text { Sistema } \\
\text { lacustre }\end{array}$ & Voucher \\
\hline L. octovalvis (Jacq.) P.H. Raven & A & erva & 1,2 & 121617 \\
\hline L. cf. quadrangularis (Micheli) H. Hara & $\mathrm{Em}$ & liana & 1,2 & 121903 \\
\hline L. sedoides (Bonpl.) H. Hara & FF & erva & 1,2 & 121792 \\
\hline L. tomentosa (Cambess.) H. Hara & $\mathrm{A} / \mathrm{Eb}$ & arbusto & 2 & 126503 \\
\hline L. torulosa (Arn.) H. Hara & $\mathrm{Em} / \mathrm{Eb}$ & arbusto & 1,2 & 121646 \\
\hline \multicolumn{5}{|l|}{ ORCHIDACEAE } \\
\hline Aspidogyne longicornu (Cogn.) Garay & Em & erva & 1 & 52064 \\
\hline Habenaria nabucoi Ruschi & Ep & erva & 1,2 & 121911 \\
\hline Platythelys debilis (Lindl.) Garay & $\mathrm{Eb}$ & erva & 2 & 131320 \\
\hline \multicolumn{5}{|l|}{ OROBANCHACEAE } \\
\hline Melasma melampyroides (Rich.) Pennell & $\mathrm{Eb}$ & erva & 2 & 121587 \\
\hline \multicolumn{5}{|l|}{ PHYLLANTHACEAE } \\
\hline Phyllanthus stipulatus (Raf.) G.L. Webster & Em & erva & 1,2 & 121704 \\
\hline \multicolumn{5}{|l|}{ PIPERACEAE } \\
\hline Piper arboreum Aubl. & Em & árvore & 2 & 121598 \\
\hline \multicolumn{5}{|l|}{ PLANTAGINACEAE } \\
\hline Bacopa cf. lanigera Wettst. & A & erva & 1 & 121867 \\
\hline B. stricta (Schrad.) Edwall & $\mathrm{Eb}$ & erva & 1,2 & 121591 \\
\hline \multicolumn{5}{|l|}{ PLANTAGINACEAE } \\
\hline Conobea scoparioides (Cham. \& Schltdl.) Benth. & A & erva & 1 & 121812 \\
\hline Scoparia dulcis $\mathrm{L}$. & A & erva & 1,2 & 121621 \\
\hline Torenia thouarsii (Cham. \& Schltdl.) Kuntze & $\mathrm{A} / \mathrm{Eb}$ & erva & 1,2 & 121588 \\
\hline \multicolumn{5}{|l|}{ POACEAE } \\
\hline Acroceras zizanioides (Kunth) Dandy & $\mathrm{A} / \mathrm{Eb}$ & erva & 1,2 & 121719 \\
\hline Andropogon bicornis L. & $\mathrm{A} / \mathrm{Eb}$ & erva & 1,2 & 121673 \\
\hline Cyphonanthus discrepans (Doll) Zuloaga \& Morrone & A & erva & 1 & 121893 \\
\hline Hymenachne amplexicaulis (Rudge) Nees & $\mathrm{Em}$ & erva & 2 & 121613 \\
\hline H. pernambucensis (Spreng.) Zuloaga & $\mathrm{A} / \mathrm{Eb}$ & erva & 1,2 & 127417 \\
\hline Leersia hexandra Sw. & A & erva & 2 & 121625 \\
\hline Megathyrsus maximus (Jacq.) B.K. Simon \& S.W.L. Jacobs & A & erva & 1,2 & 121844 \\
\hline Panicum mertensii Roth & $\mathrm{Eb}$ & erva & 1 & 52043 \\
\hline P. schwackeanum $\mathrm{Mez}$ & $\mathrm{A} / \mathrm{Eb}$ & erva & 1,2 & 121639 \\
\hline Panicum sp. & A & erva & 1 & 127339 \\
\hline Paspalum commutatum Nees & $\mathrm{A} / \mathrm{Eb}$ & erva & 1,2 & 121610 \\
\hline P. densum Poir. & A & erva & 2 & 121550 \\
\hline Paspalum sp. & Em & erva & 1 & 121891 \\
\hline Saccharum asperum (Nees) Steud. & $\mathrm{A} / \mathrm{Eb}$ & erva & 1,2 & 121689 \\
\hline Sacciolepis vilvoides (Trin.) Chase & Em & erva & 2 & 127393 \\
\hline Steinchisma laxa (Sw.) Zuloaga & $\mathrm{A} / \mathrm{Eb}$ & erva & 1,2 & 121549 \\
\hline Urochloa sp. & $\mathrm{A} / \mathrm{Eb}$ & erva & 2 & 121566 \\
\hline \multicolumn{5}{|l|}{ POLYGONACEAE } \\
\hline Polygonum acuminatum Kunth & $\mathrm{A} / \mathrm{Eb}$ & erva & 1,2 & 121702 \\
\hline P. meissnerianum Cham. \& Schltdl. & $\mathrm{A} / \mathrm{Eb}$ & erva & 1,2 & 121700 \\
\hline P. persicaria $\mathrm{L}$. & A & erva & 1,2 & 121740 \\
\hline P. punctatum Elliot & $\mathrm{A}$ & erva & 1 & 32824 \\
\hline \multicolumn{5}{|l|}{ PONTEDERIACEAE } \\
\hline Eichhornia azurea (Sw.) Kunth & $\mathrm{FF}$ & erva & 1,2 & 121682 \\
\hline
\end{tabular}




\begin{tabular}{|c|c|c|c|c|}
\hline Grupo taxonômico & $\begin{array}{c}\text { Forma } \\
\text { biológica }\end{array}$ & Hábito & $\begin{array}{l}\text { Sistema } \\
\text { lacustre }\end{array}$ & Voucher \\
\hline \multicolumn{5}{|l|}{ RUBIACEAE } \\
\hline Borreria sp. & $\mathrm{Eb}$ & erva & 1 & 121824 \\
\hline Diodia saponariifolia (Cham. \& Schltdl.) K. Schum. & A & erva & 1 & 131702 \\
\hline D. sarmentosa $\mathrm{Sw}$ & $\mathrm{A} / \mathrm{Eb}$ & erva & 1,2 & 121731 \\
\hline Diodia sp. & $\mathrm{Eb}$ & erva & 1 & 121703 \\
\hline Palicourea marcgravii A. St.-Hil. & $\mathrm{Eb}$ & arbusto & 1 & 131695 \\
\hline \multicolumn{5}{|l|}{ TYPHACEAE } \\
\hline Typha domingensis Pers. & $\mathrm{A} / \mathrm{Eb}$ & erva & 1,2 & 121660 \\
\hline \multicolumn{5}{|l|}{ URTICACEAE } \\
\hline Boehmeria cylindrica (L.) Sw. & $\mathrm{A} / \mathrm{Eb}$ & erva & 1,2 & 121768 \\
\hline Cecropia pachystachya Trec. & $\mathrm{A} / \mathrm{Eb}$ & árvore & 1,2 & 121662 \\
\hline \multicolumn{5}{|l|}{ VERBENACEAE } \\
\hline Lantana camara $\mathrm{L}$. & $\mathrm{Eb}$ & arbusto & 2 & 29507 \\
\hline Phyla betulifolia (Kunth) Greene & A & erva & 2 & 121539 \\
\hline \multicolumn{5}{|l|}{ VITACEAE } \\
\hline Cissus erosa Rich. & $\mathrm{A} / \mathrm{Eb}$ & liana & 1,2 & 121537 \\
\hline C. verticillata (L.) Nicolson \& C.E. Jarvis & $\mathrm{Eb}$ & liana & 1 & 121841 \\
\hline \multicolumn{5}{|l|}{ XYRIDACEAE } \\
\hline Xyris jupicai Rich. & $\mathrm{A} / \mathrm{Eb}$ & erva & 1,2 & 121536 \\
\hline
\end{tabular}

plasticidade fenotípica, elevadas taxas de crescimento vegetativo e dispersão de propágulos em longas distâncias (Santamaría 2002). Tal fato se reflete nos inventários que tratam de vegetais aquáticos no Brasil e no mundo (Cook et al. 1974; Pott \& Pott 2000; Bove et al. 2003; Amaral et al. 2008, entre outros) que, via de regra, apresentam plantas amplamente distribuídas, sobretudo relacionadas às formas biológicas submersas e emergentes.

Nesta pesquisa, as formas biológicas de macrófitas aquáticas descritas na literatura (Irgang et al. 1984; Pedralli 1990) foram levantadas. Entretanto, o esquema desses autores não inclui plantas estabelecidas em solos orgânicos de ilhas flutuantes (histossolos) que, peculiarmente, são comuns na flora aquática do Vale do Rio Doce. Devido a essa lacuna na literatura sobre a classificação das formas biológicas, Pivari et al. (2008a) optaram por expandir a concepção da forma epífita para o caso das espécies fixas ao histossolo dos baceiros no Pantanal. Porém, visando um melhor discernimento sobre a forma de ocorrência dos vegetais aquáticos, a partir deste trabalho os autores sugerem a utilização do termo embalsada (Neiff 1982) para se referir a uma nova categoria de formas biológicas (Fig. 2), contemplando as macrófitas aquáticas enraizadas em solos orgânicos de ilhas flutuantes (Duzer 2004). Assim, as macrófitas aquáticas do conjunto de lagoas e brejos do Vale do Rio Doce foram classificadas em anfíbias (95 spp.), embalsadas (85), emergentes (28), epífitas (3), flutuantes fixas (9), flutuantes livres (2), submersas fixas (5), submersas livres (4). O número de espécies citado acima supera o total das registradas nesse inventário, pois parte delas (47 spp.) foi enquadrada como ocorrente em duas categorias (Tab. 1).

Segundo Pedralli (2000), espécies anfíbias e emergentes são predominantes em relação às outras formas biológicas em Minas Gerais. Todavia, no conjunto de lagos e brejos do Vale do Rio Doce destacou-se a presença de embalsadas, muitas exclusivamente pertencentes a tal forma biológica (37 spp.). Essa característica, já demonstrada por Pivari $e t$ al. (2008b) em uma das lagoas pertencente ao sistema lacustre, esteve relacionada ao surgimento de ilhas flutuantes. Tais massas de vegetação foram observadas, sobretudo, em ambientes aquáticos com maior grau de eutrofização, fator possivelmente relacionado à supressão da mata no entorno dos mesmos (Tundisi \& Meis 1985). Tais ambientes são encontrados prioritariamente na zona de amortecimento do PERD e demonstram reunir condições favoráveis à sucessão avançada das plantas aquáticas, que culmina em ilhas flutuantes (Pott \& Pott 2003; Pivari et al. 2008a). 
Atualmente, cerca de 110 ambientes aquáticos do sistema lacustre do Vale do Rio Doce (mais de dois terços do total) encontram-se em áreas não protegidas (Fig. 1), onde vem sendo registradas perdas na biodiversidade relacionadas a diferentes intervenções antrópicas (Latini et al. 2004). Avaliar a ocorrência das espécies de macrófitas aquáticas no sistema lacustre como um todo, através de uma caracterização da similaridade florística entre essa área e aquela protegida pelo PERD, pode subsidiar um melhor entendimento acerca da conservação dessa vegetação na região.

A aplicação do índice de Jaccard demonstrou haver uma similaridade florística média $\left(S_{i}=0,55\right)$ entre áreas preservadas (inseridas no PERD) e não preservadas (localizadas na zona de amortecimento). Considerando o total de ambientes aquáticos amostrados, tal valor sugere que o PERD representa um importante refúgio para a maior parte das espécies: 101 em comum com a zona de amortecimento e 35 exclusivas. Entretanto, destacou-se a presença de 48 espécies (26\% do total) ocorrentes somente na zona de amortecimento, dentre as quais podem ser citadas, Bactris setosa, Echinodorus macrophyllus, Hydrocleys nymphoides, Leptostelma maximum, Ludwigia tomentosa, Nymphaea amazonum, $N$. ampla, Phyla betulifolia, Platythelys debilis, Thalia geniculata e Utricularia poconensis. O fato de parte das espécies de macrófitas aquáticas não ocorrer em áreas protegidas, provavelmente, está vinculado às lagoas e brejos desse sistema lacustre apresentaremse como unidades isoladas em relação ao estabelecimento de organismos aquáticos, como apresentado por Rebouças et al. (2006).

Nesse contexto, em um sistema lacustre que apresenta tais proporções, compreender a distribuição das espécies de macrófitas aquáticas torna-se importante para definir estratégias de conservação de toda a comunidade, visto que o total de ambientes aquáticos atualmente protegido não demonstra ser suficiente para tal. Pesquisas complementares envolvendo caracterizações dos ambientes aquáticos individualmente abordando a composição florística e padrões de distribuição das espécies no mosaico de lagoas e brejos podem contribuir com a indicação de áreas localizadas na zona de amortecimento do PERD úteis à manutenção de macrófitas aquáticas na região.

\section{Agradecimentos}

Os autores agradecem a Geovane Tavares, Marcos Silva, Higor Magalhães, Rafael Cortat, Alex Chavier, Marcos Antônio Pivari, Antônio Veloso,

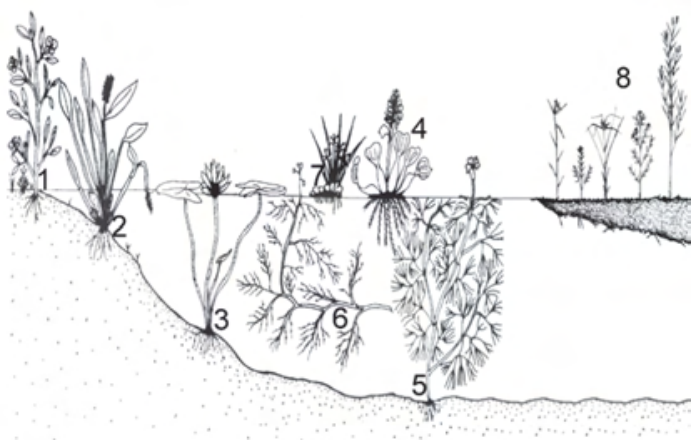

Figura 2 - Esquema de macrófitas aquáticas adaptado de Pott \& Pott (2000) a partir das formas biológicas de Irgang et al. (1984). Legenda: 1 - anfíbia, 2 - emergente, 3 - flutuante fixa, 4 - flutuante livre, 5 - submersa fixa, 6 - submersa livre, 7 - epífita e 8 - embalsada (planta enraizada em histossolo de ilha flutuante).

Figure 2 - Aquatic macrophytes scheme adapted from Pott \& Pott (2000) based in life forms from Irgang et al. (1984). Label: 1 - amphibian, 2 - emerging, 3 - fixed floating, 4 - free floating, 5 - fixed submerged, 6 - free submerged, 7 - epiphyte and 8 "embalsada" (plant established on floating meadows).

Vinícius Dittrich, Jacinto Lana e Jaílson de Oliveira, a ajuda nas expedições a campo, aos taxonomistas Vali Pott (diversos táxons), Pedro Viana (Poaceae), Nara Mota (Xyridaceae), Aristônio Teles, Erick Hattori e Jimi Nakajima (Asteraceae), Thaís Almeida (Lomariopsidaceae), Fátima Salimena (Verbenaceae), e Rosana Romero (Melastomataceae), o auxílio nas identificações botânicas. A João Renato Stehmann, Philippe Maillard, Sylvia Meyer e Arnildo Pott, a revisão do manuscrito e à Thaiz Amaral, a confecção do mapa. Finalmente, à FAPEMIG e à CAPES, o apoio financeiro.

\section{Referências}

Amaral, M.C.E.; Bittrich, V.; Faria, A.D.; Anderson, L.O. \& Aona, L.Y.S. 2008. Guia de campo para plantas aquáticas e palustres do estado de São Paulo. Holos Editora, Ribeirão Preto. 452p.

APG III - Angiosperm Phylogeny Group. 2009. An update of the Angiosperm Phylogeny Group classification for the orders and families of flowering plants: APG III. Botanical Journal of the Linnean Soviety. 17p.

Bove, C.P.; Gil, A.S.B.; Moreira, C.B. \& Anjos, R.F.B. 2003. Hidrófitas fanerogâmicas de ecossistemas aquáticos temporários da planície costeira do estado do Rio de Janeiro, Brasil. Acta Botanica Brasilica 17: 119-135.

Brummitt, R.K. \& Powell, C.E. 1992. Authors of plant names. Royal Botanic Gardens, Kew. 732p. 
Ceska, A. \& Ceska, O. 1986. More on the techniques for collecting aquatic and marsh plants. Annals of Missouri Botanical Garden 73: 825-827.

Cook, C.D.K.; Gut, B.J.; Rix, E.M. ; Schneller, J. \& Seitz, M. 1974. Water plants of the world: a manual for the identification of the genera of freshwater macrophytes. W. Junk, The Hague. 561p.

Duzer, C.V. 2004. Floating islands - A global bibliography with an edition and translation of G.C. Munz's Exercitatio academica de insulis natantibus (1711). Cantor Press, Los Altos Hills. 404p.

Esteves, F.A. 1988. Fundamentos de limnologia. $2^{\text {a }}$ ed. Interciência, Rio de Janeiro. 602p.

Haynes, R.R. 1984. Techniques for collecting aquatic and marsh plants. Annals of Missouri Botanical Garden 71: 229-231.

Irgang, B.E.; Pedralli, G. \& Waechter, J.I. 1984. Macrófitos aquáticos da Estação Ecológica do Taim, Rio Grande do Sul, Brasil. Roessleria 6: 395-404.

Junk, W.J. \& Silva, C.J. 1999. O conceito do pulso de inundação e suas implicações para o Pantanal de Mato Grosso. In: Anais do II Simpósio sobre Recursos Naturais e Sócio-Econômicos do Pantanal. Manejoe Conservação. EMBRAPA-DDT, Corumbá. Pp. 17-28

Kramer K.U. \& Green P.S. 1990. The families and genera of vascular plants. Pteridophytes and gymnosperms. Vol. 1. Springer Verlag, New York. 404p.

Latini, A.O.; Lima-Junior, D.P.; Giacomini, H.C.; Latini, R.O.; Resende, D.C.; Espírito-Santo, H.M.V.; Barros, D.F. \& Pereira, T.F. 2004. Alien fishes in lakes of the Doce river basin (Brazil): range, new occurrences and conservation of native communities. Lundiana 5: 135-142.

Maia-Barbosa, P.M.; Barbosa, L.G.; Brito, S.L.; Garcia, F.; Barros, C.F.A.; Souza, M.B.G.; Mello, N.; Guimarães, A.S. \& Barbosa, F.A.R. 2010. Limnological changes in Dom Helvécio Lake (SouthEast Brazil): natural and anthropogenic causes. Brazilian Journal of Biology 70: 795-802.

Medeiros, R. 2006. Evolução das tipologias e categorias de áreas protegidas no Brasil. Ambiente e Sociedade 9: 41-64.

Neiff, J.J. 1982. Esquema sucesional de la vegetacion em islas flotantes del chaco argentino. Boletin de la Sociedad Argentina de Botánica 21: 325-341.

Pedralli, G. 1990. Macrófitos aquáticos: técnicas e métodos de estudos. Estudos de Biologia 26: 5-24.

Pedralli, G. 2000. Padrões florísticos como subsídios à conservação da biodiversidade de macrófitas aquáticas. In: Tópicos Atuais em Botânica. EMBRAPA, Brasília. Pp. 335-339.

Pivari, M.O.D.; Pott, V.J. \& Pott, A. 2008a. Macrófitas aquáticas de ilhas flutuantes (baceiros) nas subregiões do Abobral e Miranda, Pantanal, MS, Brasil. Acta Botanica Brasilica 22: 559-567.
Pivari, M.O.D.; Salimena, F.R.; Pott, V.J. \& Pott, A. 2008b. Macrófitas aquáticas da Lagoa Silvana, Vale do Rio Doce, Minas Gerais, Brasil. Iheringia Série Botânica 63: 321-327.

Pott, V.J. \& Pott, A. 2000. Plantas aquáticas do Pantanal. EMBRAPA, Corumbá. 353p.

Pott, V.J. \& Pott, A. 2003. Dinâmica da vegetação aquática do Pantanal. In: Thomaz, S.M. \& Bini, L.M. (eds.). Ecologia e manejo de macrófitas aquáticas. EdUEM, Maringá. Pp. 145-162.

Raven, H.P.; Evert, R.F. \& Eichhorn, S.E. 1996. Biologia Vegetal. Guanabara Koogan, Rio de Janeiro. 906p.

Rebouças, A.C.; Braga, B.P.F. \& Tundisi, J.G. 2006. Águas doces no Brasil: capital ecológico, uso e conservação. $3^{a}$ ed. Escrituras, São Paulo. 748p.

Saijo, Y.\& Tundisi, J.G. 1985. Limnological studies in central Brazil - Rio Doce Valley lakes and Pantanal wetland (1st Report). Water Research Institute, Nagoya. 201p.

Saijo, Y.; Tundisi, J.G. 1989. Limnological studies in Rio Doce Valley lakes Brazil (3 ${ }^{\text {rd }}$ Report). Water Research Institute, Nagoya. 159p.

Santamaría, L. 2002. Why are most aquatic plants widely distributed? Dispersal, clonal growth and small-scale heterogeneity in a stressful environment. Acta Oecologica 23: 137-154.

SMASP - Secretaria do Estado do Meio Ambiente do Estado de São Paulo. 1997. Convenção de Ramsar sobre zonas úmidas de importância internacional, especialmente como habitat de aves aquáticas. Secretaria do Meio Ambiente, São Paulo. 24p.

Teles, A.M.; Sobral, M. \& Nakajima, J.M. 2010. A new species of Lepidaploa (Vernonieae - Asteraceae) from southeastern Brazil. Rodriguésia 61: 101-103.

Thomaz, S.M. 2006. Efeito de fatores limnológicos sobre a colonização e estrutura de assembléias de macrófitas aquáticas. In: Anais do $57^{\circ}$ Congresso Nacional de Botânica. $13^{\circ}$ Encontro Estadual de Botânicos e $5^{\circ}$ Encontro Estadual de Herbários. Imagine Designe Editorial, Gramado. Pp. 501-505.

Thomaz, S.M \& Bini, L.M. 2003. Análise crítica dos estudos sobre macrófitas aquáticas desenvovidos no Brasil. In: S.M. Thomaz \& L.M. Bini (eds.). Ecologia e manejo de macrófitas aquáticas. Editora da Universidade Estadual de Maringá, Maringá. Pp. 19-38.

Tundisi, J.G.; Matsumura-Tundisi, T.; Pontes, M.C.F. \& Gentil, J.G. 1981. Limnological studies at quaternary lakes in eastern Brazil. I. Primary production of phytoplankton and ecological factors at lake D. Helvecio. Revista Brasileira de Botânica 4: 5-14.

Tundisi, J.G. \& Meis, M.R.M. 1985. Geomorphology and limnological processes at the middle Rio Doce valley lakes. In: Saijo, Y. \& Tundisi, J.G. (ed.). Limnological studies in central Brazil - Rio Doce Valley lakes and Pantanal Wetland ( $1^{\text {st }}$ Report). Water Research Institute, Nagoya. Pp. 11-20. 